\title{
Otimização do processo de extração taninos de Pinus oocarpa para manufatura de adesivos para madeira
}

\author{
Optimization of the Pinus oocarpa tannin extraction \\ process on process for the manufactures pf wood adhesives
}

\author{
Nayara Dorigon Rodrigues ${ }^{1}$, Roberto Carlos Costa Lelis ${ }^{2}$, \\ Alexandre Miguel do Nascimento ${ }^{2}$, Michel Cardoso Vieira ${ }^{3}$ e Jéssica Chaves Cardoso ${ }^{4}$
}

\begin{abstract}
Resumo
Esse trabalho teve por objetivo verificar a influência da temperatura e do teor de sal extrator na extração de taninos condensáveis da casca de Pinus oocarpa para o uso na colagem da madeira. Um experimento fatorial composto por três níveis de temperatura $\left(60^{\circ} \mathrm{C}, 80^{\circ} \mathrm{C}, 100^{\circ} \mathrm{C}\right)$ e 4 níveis de concentração de sulfito de sódio $(0 \%, 1 \%, 3 \%, 5 \%)$ foi utilizado. A determinação das propriedades dos extratos tânicos foi realizada através da obtenção dos teores de polifenóis condensados pelo método de Stiasny (Número de Stiasny - NS), teores de taninos, teores de não-taninos e valor $\mathrm{pH}$. Os resultados mostraram que o tratamento com $5 \%$ de sulfito de sódio à $100^{\circ} \mathrm{C}$, apresentou o maior rendimento em taninos condensáveis $(17 \%)$, sendo este favorecido pela temperatura mais elevada.
\end{abstract}

Palavras-chave: casca, Temperatura, polifenois, sulfito de sódio.

\begin{abstract}
This study investigates the influence of temperature and salt extractor content on the extraction of condensable tannins from Pinus oocarpa bark, to be used in bonding wood. A factorial design consisting of three temperature levels $\left(60^{\circ} \mathrm{C}, 80^{\circ} \mathrm{C}, 100^{\circ} \mathrm{C}\right)$ and four concentration levels of sodium sulfite $(0 \%, 1 \%$, $3 \%, 5 \%$ ) was used. For tannin extracts, the properties determined were: condensed polyphenol content (Stiasny methods); tannin; non- tannin content and $\mathrm{Ph}$ value. The results showed that the treatment with $5 \%$ sodium sulfite at $100^{\circ} \mathrm{C}$, resulted in the highest yield of condensed tannins (17\%); this being favored by higher temperature.
\end{abstract}

Keywords: bark, temperature, polyphenols, sodium sulfite.

\section{INTRODUÇÃO}

A destinação da casca é um problema bastante complexo para as indústrias de conversão de madeira, visto o grande volume gerado durante o processamento de toras. As cascas por sua vez possuem em sua composição química polifenóis como os taninos que há anos são estudados como substâncias potenciais para colar madeira (ROFFAEL; DIX, 1994). A utilização das cascas para extração de taninos na produção de adesivos para colagem de madeira é uma opção economicamente e ambientalmente interessante diante da problemática de destinação destes resíduos.

Entretanto, Gonçalves (2000) afirmou que a qualidade da colagem é influenciada pelos produtos químicos utilizados na extração dos taninos. O mesmo foi observado por Panamgama (2007), o qual atentou para o fato de que nem sempre os maiores rendimentos em taninos significam uma melhor qualidade na colagem.

Os diferentes processos de extração influenciam diretamente na obtenção de taninos a baixo preço, de alta qualidade e em quantidades suficientes para a substituição efetiva dos materiais poliméricos na manufatura de adesivos para madeira (PING et al., 2011).

\footnotetext{
${ }^{1}$ Mestranda em Ciências Ambientais e Florestais. UFRRJ - Universidade Federal Rural do Rio de Janeiro/ Instituto de Florestas. BR 465 Km 07 - 23890-000 - Seropédica - RJ. E-mail: nayaradorirodrigues@yahoo.com.br

${ }^{2}$ Professor Associado - UFRRJ - Universidade Federal Rural do Rio de Janeiro - Instituto de Florestas - Departamento de Produtos Florestais - BR 465, Km 07 - CEP: 23890-000 - Seropédica, RJ - E-mail: lelis@ufrri.br; amn@ufrrj.br

${ }^{3}$ Doutorando em Ciências Ambientais e Florestais. UFRRJ - Universidade Federal Rural do Rio de Janeiro / Instituto de Florestas. BR 465, Km 07 - 23890-000 - Seropédica, RJ. E-mail: mceflorestal@ig.com.br

${ }^{4}$ Graduanda em Engenharia Florestal. UFRRJ - Universidade Federal Rural do Rio de Janeiro / Instituto de Florestas. BR 465, Km 07 - 23890-000 - Seropédica, RJ. E-mail: jessicachavescardoso@yahoo.com.br
}

Sci. For., Piracicaba, v. 43, n. 108, p. 1013-1020, dez. 2015

DOI: dx.doi.org/10.18671/scifor.v43n108.24 
Nos processos mais comuns de extração de taninos são utilizados sais como solvente em solução aquosa sob aquecimento. Os principais sais utilizados para extração de taninos com a finalidade de melhorar a qualidade e aumentar os rendimentos são o sulfito de sódio $\left(\mathrm{Na}_{2} \mathrm{SO}_{3}\right)$, o carbonato de sódio $\left(\mathrm{Na}_{2} \mathrm{CO}_{3}\right)$, o hidróxido de sódio $(\mathrm{NaOH})$, o dissulfito de sódio $\left(\mathrm{Na}_{2} \mathrm{~S}_{2} \mathrm{O} 5\right)$ e o bissulfito de sódio $\left(\mathrm{NaHSO}_{3}\right.$ ) (PIZZI; MITTAL, 1994; SILVA, 2001).

A temperatura é um fator relevante que influencia na extração de tanino da casca das árvores. Ping et al. (2011), estudando a influência das temperaturas de 70,100 e $120^{\circ} \mathrm{C}$ na extração de taninos do bagaço de uva, observaram que o aumento da temperatura de processo e da concentração dos reagentes resultou em uma redução do teor de açúcares. Entretanto, os adesivos produzidos com tanino extraídos a $120^{\circ} \mathrm{C}$ apresentaram uma menor reatividade junto ao formaldeído, e os adesivos produzidos de taninos extraídos em condições mais brandas, $70^{\circ} \mathrm{C}$ apresentaram menor solubilidade em água devido à solubilização de taninos de maior massa molecular Logo observa-se que a temperatura é um fator que apresenta relação com a eficiência do processo de extração de taninos, e que a temperatura mais eficiente varia de espécie para espécie, sendo relevante os estudos para definição de temperaturas de extração de taninos eficientes para o uso dos taninos como adesivo de madeira.

Estudos anteriores revelaram que a espécie Pinus oocarpa destaca-se por apresentar alto rendimento na extração de taninos de sua casca (FERREIRA, 2009; VIEIRA, 2011). Entretanto, o conhecimento sobre o processo de extração de taninos da casca e as suas propriedades adesivas é ainda incipiente.

Dessa forma, o presente trabalho teve por objetivo estudar o efeito dos diferentes níveis de sulfito de sódio na extração de tanino da casca de Pinus oocarpa em diferentes níveis de temperatura e aprofundar os conhecimentos sobre o processo de extração.

\section{MATERIAL E MÉTODOS}

\section{Descrição do Material}

Para coleta das cascas, cinco árvores de P.oocarpa com idade de 31 anos e com diâmetro à altura do peito (DAP) variando entre 15 e 40 centímetros foram escolhidas aleatoriamente de povoamento florestal na cidade de Estrela do Sul - MG. As cascas foram retiradas manualmente do DAP até a base das árvores em pé, de modo a padronizar a amostragem.

\section{Extração dos Taninos}

A casca, fragmentada em moinho de martelo, passou pelo processo de extração, usando-se água pura e com adição de diferentes percentuais de sulfito de sódio $(0,1,3$ e 5\%) em três diferentes temperaturas $\left(60,80\right.$ e $\left.100^{\circ} \mathrm{C}\right)$. O material foi extraído em balão sob refluxo por 2 horas, utilizando-se uma relação licor/casca de 15:1. Após a extração, o material foi filtrado a vácuo, sendo separado o material filtrado para análise.

\section{Determinação das Propriedades dos Extratos Tânicos}

Para determinação dos teores de extrativos, uma alíquota de $25 \mathrm{ml}$ do liquido extraído foi colocada em uma placa de petri, previamente tarada, e levada em estufa à $103^{\circ} \pm 2^{\circ} \mathrm{C}$, até peso constante. Da diferença entre a massa da placa de petri antes e depois de ser levada à estufa com a alíquota, obtém-se a quantidade de extrativos (g) em $25 \mathrm{ml}$ de solução e considerando-se a quantidade de partículas (base seca) e o volume inicial empregados na extração, calcula-se o teor de extrativos em percentagem.

O cálculo do teor de polifenóis condensados foi realizado pelo método do número de Stiasny (NS) baseado na metodologia de WISSING (1955); LELIS (1995).

A obtenção do percentual de tanino e não-taninos na casca foi realizada de acordo com Vieira et al. (2011) onde para a obtenção do percentual de tanino na casca multiplica-se o número de Stiasny pelo teor de extrativos totais determinado e converte-se o resultado em percentagem. A diferença entre teor de extrativo e tanino fornece a percentagem de não-taninos. 


\section{Delineamento experimental e Análise estatística}

O delineamento experimental foi um fatorial, sendo utilizados como fatores os diferentes teores de sulfito de sódio (ausência, 1\%, 3 e $5 \%)$ e as diferentes temperaturas $\left(60^{\circ} \mathrm{C}, 80^{\circ}\right.$ e $100^{\circ} \mathrm{C}$ ) usadas no processo de extração, totalizando - se assim 12 tratamentos.

Para cada tratamento, foram realizadas extrações em que se determinou dos extratos obtidos, as seguintes propriedades, a saber: teor de extrativo total, teor de polifenóis condensados, teor de taninos, teor de não-taninos e valor de $\mathrm{pH}$.

Para avaliar os resultados, foi empregada uma analise de variância (ANOVA). Para tal, os pressupostos da ANOVA, normalidade dos dados e homogeneidade das variâncias, das variáveis dependentes foram testados pelo método de Kolmogorov-Smirnov e Levene, respectivamente, em nível de $5 \%$ significância. Foi considerado na analise de variância o efeito dos fatores principais e a interação entre os fatores. Quando valores de F foram significativos, comparações entre médias foram feitas por meio de testes de médias de Tukey a 1 e $5 \%$ significância.

\section{RESULTADOS E DISCUSSÃO}

\section{Resultados obtidos pela ANOVA fatorial}

Apesar de serem valores percentuais, o teor de extrativo total, o teor de polifenol condensado e os percentuais de taninos e não taninos apresentaram distribuição normal e variâncias homogêneas. Em todas as análises de variâncias, o efeito da temperatura, do teor de sulfito de sódio e da interação entre os fatores foram significativos. O mesmo ocorreu com a variável $\mathrm{pH}$.

\section{Teor de extrativos totais}

Observa-se na Tabela 1 que o aumento do teor de sulfito de sódio acarretou no aumento do teor de extrativos totais em todos os diferentes niveis de temperatura testados. Quando se avalia o efeito da temperatura independentemnte do teor de sulfito, observa-se um aumento signficativo da extração da casca de Pinus oocarpa.

Diversos trabalhos encontrados na literatura comprovam o aumento do teor de extrativos, quando se emprega um sal extrator (VITAL et al., 2004; FERREIRA et al., 2009; CARNEIRO, 2010; VIEIRA et al., 2011), o sulfito de sódio através do processo de sulfitação aumentou a solubilização dos taninos em água.

Segundo Silva (2001) a sulfitação aumenta a solubilidade do tanino em água a medida que ocorre a eliminação do grupamento éter no anel heterocíclico das moléculas de taninos, este anel é hidrofóbico além de que os taninos quimicamente não se constituem em soluções verdadeiras, mas em suspensões hidrocoloidais, em que parte das moléculas de taninos se hidrata, enquanto outra parte tende a repelir a hidratação; logo a introdução de um grupamento sulfônico e uma hidroxila nas unidades flavanóides dos taninos proporciona um aumento de seu caráter hidrofílico favorecendo a solubilização em agua e aumentando os rendimentos em taninos.

Tabela 1. Comparações múltiplas entre os valores médios do teor de extrativos totais da casca de Pinus oocarpa, obtidos em diferentes percentagens de sulfito de sódio e diferentes temperaturas.

Table 1. Mean values and comparisons between means for the variable content of total extractives of Pinus oocarpa bark, in different percentages of sodium sulfite and different temperatures.

\begin{tabular}{|c|c|c|c|c|}
\hline \multirow[b]{2}{*}{ Teor de Sulfito de Sódio - \% } & \multicolumn{3}{|c|}{ Temperatura de Extração $-^{\circ} \mathrm{C}$} & \multirow[b]{2}{*}{ Médias } \\
\hline & $60^{\circ} \mathrm{C}$ & $80^{\circ} \mathrm{C}$ & $100^{\circ} \mathrm{C}$ & \\
\hline 0 & $3,90_{(0,85)} \mathrm{dB}$ & $7,23_{(0,89)} \mathrm{dA}$ & $8,53_{(1,32)} d A$ & $6,55 \mathrm{~d}$ \\
\hline 1 & $8,04_{(0,72)} \mathrm{cA}$ & $10,02_{(0,57)} \mathrm{cA}$ & $11,749_{(1,05)} \mathrm{cA}$ & $9,93 \mathrm{c}$ \\
\hline 3 & $10,12_{(0,71)}^{b C}$ & $13,44_{(0,71)} b B$ & $16,96_{(1,09)} b A$ & $13,51 b$ \\
\hline 5 & $13,66_{(0,67)} \mathrm{aC}$ & $16,96_{(0,70)} \mathrm{aB}$ & $20,55_{(1,57)} \mathrm{aA}$ & 17,06 a \\
\hline Médias & $8,93 \mathrm{C}$ & $11,91 \mathrm{~B}$ & $14,45 \mathrm{~A}$ & 11,76 \\
\hline
\end{tabular}

Letras minúsculas distintas diferem as médias na mesma coluna, e letras maiúsculas distintas diferem as médias na mesma linha, em nível de $5 \%$ de de probabilidade de erro pelo teste de Tukey. Valores entre parêntese são os desvios padrões.

Different lowercase letters differ from the mean in the same column, and different capital letters differ from the mean on the same row, at the $5 \%$ significance level by the Tukey test. Values in parentheses are standard deviations. 
Entretanto, quando o sulfito de sódio esta associado ao fator temperatura, os quais foram, e quanto maiores são os teores de sulfito de sódio e maiores são as temperaturas, maiores são os teores de extrativos totais obtidos. Observa-se que para $1 \%$ de sal extrato na tempertura de $60^{\circ} \mathrm{C}$ obetve-se $8,04 \%$ enquanto para $5 \%$ de sal e temperatura de $100^{\circ} \mathrm{C}$ o valor obtido foi de 20,55 , um aumento maior que $155 \%$. O aumento da quantidade de sulfito de sódio potencializou o efeito da temperatura, favorecendo a extração de extrativos totais, provavelmente pela maior dissolução das diversas especies moleculares presentes na casca dos taninos de P. oocarpa. Segundo Silva (2001), o aumento da solubilidade em água é responsável pelo maior rendimento em extrativos totais.

Ferreira et al. (2009) encontraram teores de extrativos obtidos com adição de $5 \%$ de sulfito de sódio à temperatura de $100^{\circ} \mathrm{C}$, de $32,49 \%$ da casca de Pinus oocarpa. Já Vieira et al. (2011) encontraram valores de $30,1 \%$ de extrativos totais para a mesma espécie. O valor encontrado neste trabalho foi bastante inferior, sendo de 20,55\%. Segundo Oliveira (2005), a quantidade e qualidade dos extrativos, sofrem variações nos teores das substâncias extrativas dentro de uma mesma espécie. Vários fatores também podem influenciar na quantidade do teor de extrativos totais como: idade, localização geográfica onde a espécie foi cultivada, região do tronco onde foram coletadas as cascas.

A principal justificativa apontada para o aumento dos teores de extrativos (aumento do rendimento) deve-se a hidrólise das ligações interflavonóides, açúcares e gomas hidrocoloidais e à abertura do anel heterocíclico das moléculas de taninos, o que acarreta o aumento do número de sítios sujeitos à hidratação, aumentando sua solubilidade em água (PIZZI,1994).

\section{Teor de polifenóis condensados}

Todos os tratamentos apresentaram Número de Stiasny maior que 50\% (Tabela 2), o que mostra o potencial das cascas da espécie de Pinus oocarpa como fonte de polifenóis condensáveis para produção de adesivos para madeira. Ping et al. (2011), citando Stiasny (1906), definiu o número de Stiasny como um indicador da pureza dos extratos de polifenóis. Pizzi (1994) afirmou que o NS é um parâmetro que indica a capacidade dos extrativos reagirem com o formaldeído na preparação de adesivos.

Tabela 2. Comparações múltiplas entre os valores médios do teor de polifenóis condensados-NS das cascas de Pinus oocarpa obtidos em diferentes porcentagem de sulfito de sódio e diferentes temperaturas.

Table 2. Mean values and comparisons between means for the polyphenol content of condensed-NS bark of Pinus oocarpa obtained by different percentages of sodium sulfite and different temperatures.

\begin{tabular}{|c|c|c|c|c|}
\hline \multicolumn{5}{|c|}{ Teor de polifenóis condensados - NS } \\
\hline \multicolumn{5}{|c|}{ Temperatura de Extração $-{ }^{\circ} \mathrm{C}$} \\
\hline Teor de Sulfito de Sódio - \% & $60^{\circ} \mathrm{C}$ & $80^{\circ} \mathrm{C}$ & $100^{\circ} \mathrm{C}$ & Médias \\
\hline 0 & $90,18_{(4,22)} \mathrm{aA}$ & $89,82_{(4,04)} a A$ & $78,34_{(3,32)} \mathrm{aB}$ & $86,40^{a}$ \\
\hline 1 & $79,30_{(2,30)} \mathrm{aA}$ & $73,77_{(5,69)}^{(1,07)} b B$ & $84,44_{(2,89)} \mathrm{aA}$ & $79,16 b$ \\
\hline 3 & $65,70_{(4,16)}^{(2,0)} b B$ & $75,69_{(4,48)}^{(0,0)} b A$ & $78,96_{(1,18)}^{(2,0)} \mathrm{aA}$ & $73,45 c$ \\
\hline 5 & $56,83_{(1,70)} b B$ & $77,90_{(2,65)}$ bA & $83,46_{(1,78)}$ aA & $72,73 c$ \\
\hline Médias & $73,01^{(1,0)} \mathrm{B}$ & $79,57^{(2,05)} \mathrm{B}$ & $81,48^{(1,18)} \mathrm{A}$ & 77,94 \\
\hline
\end{tabular}

Letras minúsculas diferem as médias na mesma coluna, e letras maiúsculas diferem as médias na mesma linha, em nível de $5 \%$ de probabilidade de erro pelo teste de Tukey. Valores entre parêntese são os desvios padrões.

Different lowercase letters differ from the mean in the same column, and different capital letters differ from the mean in the same line, at the $5 \%$ significance level by Tukey test. Values in parentheses are standard deviations.

Ferreira et al. (2009) e Vieira et al. (2011) encontrar valores de NS de 97,32\% e 89,9\% respectivamente para extração realizada a $100^{\circ} \mathrm{C}$ e com $5 \%$ de sulfito de sódio. Tais valores são superiores aos encontrados neste trabalho, que foi de 83,46, para as mesmas condições.

Em seu estudo, Jorge et al. (2001) encontraram uma relação inversa do aumento de temperatura da extração com o número de Stiasny ao estudarem o melhor processo de extração para Pinus pinaster, utilizando hidróxido de sódio a 3\%. O aumento da temperatura de extração tornou a extração menos específica para as substâncias condensáveis com o formaldeído, obtendo-se valores de NS de $105 \%$ para temperatura de $20^{\circ} \mathrm{C}$ e de $75 \%$ para temperaturas de $100^{\circ} \mathrm{C}$.

A influência do teor do sal extrator sulfito de sódio para os valores de NS foi mais expressiva na temperatura de $60^{\circ} \mathrm{C}$. O aumento do teor do sal extrator gerou menores valores de NS. Na temperatura de $80^{\circ} \mathrm{C}$, embora o acréscimo de sal extrator tenha gerado valores numericamente diferentes, 
esses não revelaram diferenças estatísticas, e desta forma tais valores diferem apenas da extração realizada sem sulfito de sódio (0\%).

Ao não se utilizar o sulfito de sódio, o aumento da temperatura influencia negativamente, ocorrendo a diminuição dos valores de NS. Utilizando-se de sulfito de sódio em concentrações de 3 e $5 \%$, observa-se uma influência positiva nos valores de NS, ocorrendo um aumento desses. No caso da extração a utilizando-se 5\% de sulfito de sódio, os valores variaram de 56,83 a 83,46. Para 3\% de sulfito de sódio os valores de NS tiveram uma menor variação $(65,70-78,96)$.

Ping et al. (2011), utilizando diferentes sais extratores, entre eles o sulfito de sódio em diferentes temperaturas, observaram que com o aumento dos valores de temperatura e da concentração de reagentes foram obtidos maiores valores de NS. Os autores justificam os resultados, devido à uma maior redução dos teores de açúcar.

Carneiro (2006), ao estudar as espécies Eucaliptus grandis e Anadenanthera peregrina observou que quanto maior o NS, menor é a porcentagem de substâncias não-tânicas presentes nos extratos. Segundo o mesmo autor, o decréscimo do valor de NS se deve a maior solubilização de açúcares, gomas, e hemiceluloses de baixo peso molecular, que não reagem com formaldeído na reação de Stiasny.

De modo geral, embora as extrações em água (sem a adição de sulfito) tenham apresentado os maiores valores de NS, essas apresentaram também os menores rendimentos em taninos, não sendo viável para produção de adesivos. A capacidade de um polifenol natural de obter um impacto significativo como substituto de material polimérico, para ser utilizado como adesivo depende da disponibilidade da fração de taninos de baixo preço, com alta qualidade e em escala suficiente para suprir a demanda (PING et al., 2011).

\section{Porcentagem de taninos}

Pela Tabela 3 é possível verificar que com o aumento da temperatura e do teor de sal, as porcentagens em taninos condensáveis aumentaram.

Tabela 3. Comparações múltiplas entre os valores médios dos rendimentos em taninos condensáveis das cascas de Pinus oocarpa extraído em diferentes temperaturas e diferentes porcentagens de sal extrator (sulfito de sódio).

Table 3. Mean values and comparisons between means for efficiencies of condensed tannins in the bark of Pinus oocarpa extracted at different temperatures and different percentages of extractor salt (sodium sulfite).

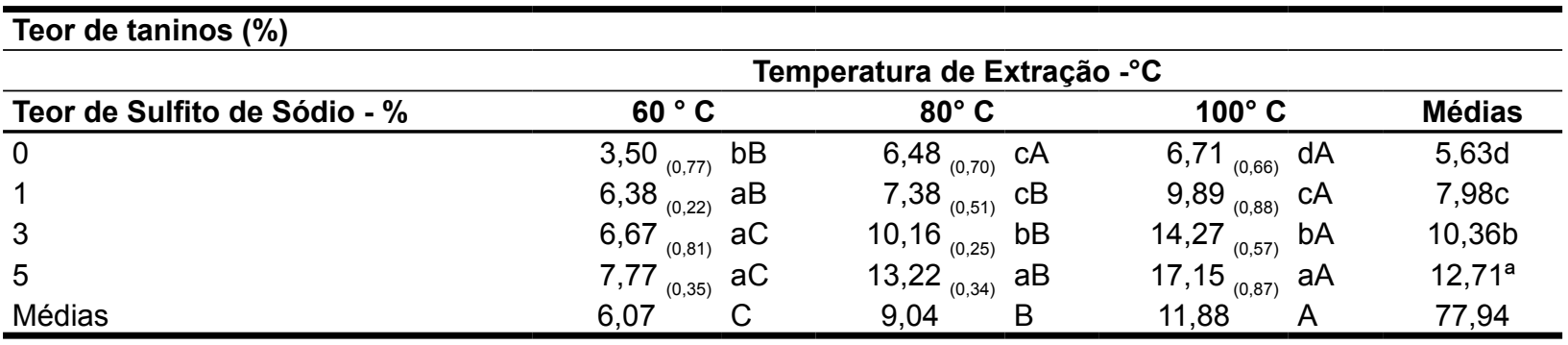

Letras minúsculas diferem as médias na mesma coluna, e letras maiúsculas diferem as médias na mesma linha, em nível de $5 \%$ de probabilidade de erro pelo teste de Tukey. Valores entre parêntese são os desvios padrões.

Different lowercase letters differ from the mean in the same column, and different capital letters differ from the mean on the same row, at $5 \%$ significance level by the Tukey test. Values in parentheses are standard deviations.

Para todos os diferentes teores de sulfito de sódio, o rendimento em taninos condensáveis aumentou à medida que a temperatura também aumentou. Foi observada uma maior variação entre os teores de taninos condensáveis para os maiores teores de sulfito de sódio em relação à temperatura. Utilizando-se 3\% de sulfito de sódio, os valores variaram entre 6,67 e 14,27 \% para as temperaturas de 60 e $100^{\circ} \mathrm{C}$, respectivamente. Esta variação para o teor de sulfito de sódio a $5 \%$ ocorreu entre 7,77 e $17,15 \%$ nas mesmas temperaturas.

O tratamento que utilizou a temperatura de $100^{\circ} \mathrm{C}$ e $5 \%$ de sulfito de sódio apresentou rendimento em taninos condensáveis de 17,15\%. Ferreira et al. (2009) e Vieira et al. (2011) encontraram valores de 27,09\% e 22,65\% respectivamente para as mesmas condições de temperatura e teor de sal.

Segundo Pizzi (1994), ao se utilizar água na extração de taninos, a temperatura de extração varia de espécie para espécie. Para acácia são utilizadas temperaturas entre 94 e $100^{\circ} \mathrm{C}$. Para as madeiras de quebracho (Schinopsis brasiliensis), pinus (Pinus sp) e pecan (Carya illinoensis) empregam-se temperaturas inferiores a $70^{\circ} \mathrm{C}$. $\mathrm{O}$ uso de altas temperaturas não melhora o rendimento em material 
fenólico e pode favorecer a extração de materiais não-fenólicos. Entretanto, os resultados obtidos nesta pesquisa demonstraram que o aumento da temperatura favoreceu a retirada dos materiais fenólicos para a espécie Pinus oocarpa.

Ping et al. (2011), ao estudar a extração de taninos condensados do bagaço de uva com adição de sulfito de sódio, comprovaram que a introdução de um grupo sulfônico por sulfitação aumenta a produção da extração de tanino e favorece a solubilidade em água; esses fatores são resultados da abertura do anel heterocíclico durante a extração.

$\mathrm{O}$ aumento da solubilidade dos taninos submetidos ao processo de sulfitação é devido ao aumento do caráter nucleofílico dos anéis " $\mathrm{A}$ " das unidades flavonóides (resorcinólicos ou floroglucinólicos), devido à introdução do grupamento sulfônico e de uma hidroxila nas unidades flavonóides, aumentando assim seu caráter hidrofílico. A sulfitação promove o decréscimo da rigidez das moléculas de taninos, do impedimento estérico e do número de ligações de hidrogênio intermoleculares, por meio da abertura do anel heterocíclico, o que acarreta aumento do numero de sítios sujeitos à hidratação (PIZZI,1983).

\section{Porcentagem de não-taninos}

Observa-se pela Tabela 4 que nos tratamentos em que não foi utilizado sal extrator (teor de sulfito de sódio $=0 \%$, o aumento da temperatura favoreceu a extração de compostos não-tânicos. $\mathrm{O}$ efeito da temperatura, analisado de forma isolada, independentemente do teor de sulfito utilizado, não teve efeito na extração de compostos não tânicos. Para os tratamentos que utilizaram teor de sulfito de sódio de 1 e 3\%, a temperatura não influenciou na extração de compostos não-tânicos.

Tabela 4. Comparações múltiplas entre os valores médios da porcentagem de não-taninos condensáveis dos extratos da casca de Pinus oocarpa obtidos em diferentes temperaturas e diferentes porcentagens de sal extrator (sulfito de sódio).

Table 4. Mean values and comparisons between means for percentages of non-condensable tannin extracts from the bark of Pinus oocarpa obtained at different temperatures and different percentages of extractor salt (sodium sulfite).

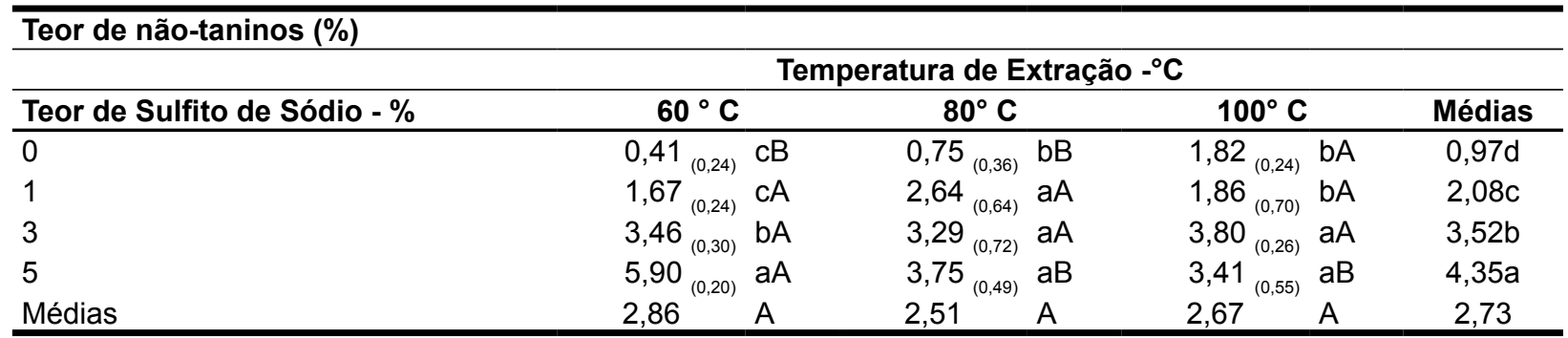

Letras minúsculas diferem as médias na mesma coluna, e letras maiúsculas diferem as médias na mesma linha, em nível de $5 \%$ de probabilidade de erro pelo teste de Tukey. Valores entre parêntese são os desvios padrões.

Different lowercase letters differ from the mean in the same column, and different capital letters differ from the mean in the same row at the $5 \%$ significance level by the Tukey test. Values in parentheses are standard deviations.

Já utilizando-se $5 \%$ de sulfito de sódio, notou-se que a $60{ }^{\circ} \mathrm{C}$ de temperatura, obtém-se a maior porcentagem de compostos não-tânicos.Segundo Ferreira et al. (2009), os não-taninos correspondem a fração de açúcares, gomas e resinas existentes nos extrativos da casca de Pinus oocarpa, e sua quantificação é importante para avaliar a qualidade dos adesivos a serem confeccionado.

Gaugler e Grigsby (2009), estudando a degradação térmica dos taninos condensados da casca de Pinus radiata afirmaram que os teores de carboidratos brutos variam em torno de 12 e 20\%, dependendo da fonte da casca da árvore e das condições de extração utilizadas.

\section{pH}

Observa-se pela Tabela 5 que o teor de sal e temperatura gera comportamentos antagônicos para o valor de $\mathrm{pH}$ dos extratos de Pinus oocarpa.

Nota-se que o aumento da temperatura favoreceu para diminuição do $\mathrm{pH}$, enquanto que o aumento do teor de sulfito de sódio favorece ao aumento dos valores de $\mathrm{pH}$. 
Tabela 5. Comparações múltiplas entre os valores médios do $\mathrm{pH}$ dos extratos de taninos da casca de Pinus oocarpa obtidos em diferentes temperaturas e diferentes porcentagens de sal extrator (sulfito de sódio).

Table 5. Mean values and comparisons between means for $\mathrm{pH}$ of tannin extracts from the bark of Pinus oocarpa obtained at different temperatures and different percentages of extractor salt (sodium sulfite).

\begin{tabular}{|c|c|c|c|c|c|c|c|}
\hline \multicolumn{8}{|l|}{$\mathrm{pH}$} \\
\hline \multirow[b]{2}{*}{ Teor de Sulfito de Sódio - \% } & \multicolumn{6}{|c|}{ Temperatura de Extração $-{ }^{\circ} \mathrm{C}$} & \multirow[b]{2}{*}{ Médias } \\
\hline & $60^{\circ} \mathrm{C}$ & & $80^{\circ} \mathrm{C}$ & & 10 & & \\
\hline 0 & $2,82_{(0,15)}$ & $\mathrm{dA}$ & $2,78_{(0,18)}$ & $\mathrm{dA}$ & $2,49_{(0,15)}$ & $\mathrm{dA}$ & $2,70 d$ \\
\hline 1 & $4,27_{(0,17)}$ & $\mathrm{cA}$ & $3,35_{(0,15)}$ & $\mathrm{cB}$ & $3,28_{(0,14)}$ & $\mathrm{cB}$ & $3,59 c$ \\
\hline 3 & $5,60_{(0,12)}$ & $\mathrm{bA}$ & $4,80_{(0,14)}$ & $\mathrm{bB}$ & $4,21_{(0,12)}$ & $\mathrm{bC}$ & $4,87 b$ \\
\hline 5 & $6,45_{(0,13)}$ & $\mathrm{aA}$ & $5,90_{(0,11)}$ & $\mathrm{aB}$ & $5,31_{(0,13)}$ & $\mathrm{aB}$ & $5,89^{a}$ \\
\hline Médias & 4,78 & A & 4,08 & $\mathrm{~B}$ & 3,79 & C & 4,26 \\
\hline
\end{tabular}

Letras minúsculas diferem as médias na mesma coluna, e letras maiúsculas diferem as médias na mesma linha, em nível de $5 \%$ de probabilidade de erro pelo teste de Tukey. Valores entre parêntese são os desvios padrões.

Different lowercase letters differ from the mean in the same column, and different capital letters differ from the mean in the same row, at $5 \%$ significance level by the Tukey test. Values in parentheses are standard deviations.

Observa-se na Tabela 5 que os valores de $\mathrm{pH}$ para extração em água pura ( $0 \%$ de sulfito de sódio) variaram entre 2,82\% e 2,49\%, os quais são valores bastante baixos e demonstram o caráter naturalmente ácido dos extrativos. Os valores médios de $\mathrm{pH}$ dos extratos obtidos, sem adição de sulfito de sódio, não foram influenciados pelas diferentes temperaturas de extração.

$\mathrm{O}$ aumento do teor de sal nas diferentes temperaturas provocou um aumento dos valores de $\mathrm{pH}$ nos extratos tânicos; isso demonstra que o pH das soluções são mais influenciados pela variação do teor de sal do que pela variação da temperatura de extração. Apenas para o teor de $3 \%$ de sulfito de sódio observou-se uma maior variação dos valores de $\mathrm{pH}$ para as diferentes temperaturas de extração diminuindo o valor do PH com o aumento da temperatura.

$\mathrm{O}$ pH encontrado para o tratamento que utilizou $5 \%$ de sulfito de sódio e temperatura de $100^{\circ} \mathrm{C}$ foi de 5,31, valor próximo aos valores encontrados por Ferreira et al. (2009) e Vieira et al. (2011) 5,42 e 5,68, respectivamente.

$\mathrm{O}$ pH é um fator importante, pois interfere no endurecimento dos adesivos (ROFFAEL; DIX, 1994). A acidez interfere na reação à medida que ocorre a condensação do tanino com formaldeído, aumentando a velocidade da reação a medida que diminui os valores de pH (VIEIRA et al., 2011). Por outro lado, condições muito alcalinas resultam em adesivos muito viscosos, não sendo possível a sua utilização como adesivo conforme foi observado por Panamgama (2007) na extração de taninos da casca de Pinus radiata em condições muito alcalinas $(\mathrm{pH}=10,5)$.

\section{CONCLUSÕES}

O aumento da temperatura $\left(60^{\circ} \mathrm{C}, 80^{\circ} \mathrm{C}, 100^{\circ} \mathrm{C}\right)$ e do teor de sulfito de sódio $(0 \%, 1 \%, 3 \%$, $5 \%$ ) favoreceram a retirada dos extrativos das cascas de Pinus oocarpa, sendo maior o efeito do sal do que o da temperatura. Há um aumento gradual da retirada dos extrativos a medida que se aumenta o nível da temperatura e o teor de sulfito de sódio.

$\mathrm{Na}$ ausência de sulfito de sódio houve as maiores extrações de polifenois enquanto que na temperatura de $100^{\circ} \mathrm{C}$ houve os maiores rendimentos.

O aumento do teor de sulfito de sódio aumenta o valor do pH dos extratos tânicos, enquanto que, o aumento do nível de temperatura diminue o valor do $\mathrm{pH}$.

O melhor processo de extração dos taninos condensáveis da casca de Pinus oocarpa dentro dos processos testados foi utilizando-se $5 \%$ de sulfito de sódio à temperatura a $100^{\circ} \mathrm{C}$.

\section{REFERÊNCIAS BIBLIOGRÁFICAS}

CARNEIRO, A. C. O. Efeito da hidrólise ácida e sulfitação de taninos de Eucalyptus grandis W.Hill ex Maiden e Anadenanthera peregrina Speg nas propriedades de adesivos. 2006. 182 p. Tese (Doutorado em Ciência Florestal) - Universidade Federal de Viçosa. Viçosa, 2006.

CARNEIRO, A. C. O.; VITAL, B. R.; CARVALHO, A. M. M. L; OLIVEIRA, A. C.; PEREIRA, B. L. C; ANDRADE, B. G. Determinação da massa molar de taninos vegetais através da técnica da cromatografia de permeação em gel. Scientia Forestalis, Piracicaba, v. 38, n. 87, p. 419-429, 2010. 
Lelis et al. - Otimização do processo de extração taninos de Pinus oocarpa para manufatura de adesivos para madeira

FERREIRA, E. S.; LELIS, R. C. C.; BRITO, E. O.; NASCIMENTO, A. M.; MAIA, J. L. S. Teores de taninos da casca de quatro espécies de pinus. Floresta e Ambiente, Seropédica, v. 16, n. 2, p. 30-39, 2009.

GAUGLER, M.; GRIGSBY, W. Thermal Degradation of Condensed Tannins from Radiata Pine Bark. Journal of Wood Chemistry and Technology, Philadelphia, v. 29, n. 4, 2009 , p. 305-321, 2009.

GONÇALVES, C. A. Utilização do tanino da madeira de Mimosa caesalpiniaefolia Bentham (Sabiá) como matéria prima alternativa para a produção de adesivos. 2000. 100 p. Dissertação (Mestrado em Ciências Ambientais e Florestais) - Universidade Federal Rural do Rio de Janeiro, Instituto de Florestas, Seropédica, 2000.

JORGE, F. C; BRITO, P; PEPINO, L.; PORTUGAL, A.; GIL, H.; COSTA, R. P. Aplicações para as Cascas de Árvores e para os Extractos Taninosos: Uma Revisão. Silva Lusitana, Lisboa, v. 9, n. 2, p. 225-236, 2001.

LELIS, R. C. C. Zur Bedeutung der Kerninhaltsstoffe obligatorisch verkernter Nadelbaumarten bei der Herstellung von feuchtbeständigen und biologisch resistenten Holzspanplatten, am Beispiel der Douglasie (Pseudotsuga menziesii Mirb. (Franco). 1995. 249 p. Tese (Doutorado em Ciências Florestais) Universität Göttingen - Göttingen, 1995.

OLIVEIRA, M. A. Extração de polifenóis da semente de Cacau (Theobroma Cacao). 2005. 72 p. Dissertação (Mestrado em Engenharia Química) - Universidade Federal de Santa Catarina, Florianópolis, 2005.

PANAMGAMA, L. A. Polyphenolic extracts of Pinus radiata Bark and networking mechanisms of additiveaccelerated polycondensates. Journal of Applied Polymer Science, v. 103, n. 4, p. 2487-2493. 2007.

PING, L.; PIZZI, A.; GUO, Z. D.; BROSSE, N. Condensed tannins extraction from grape pomace: Characterization and utilization as wood adhesives for wood particleboard. Industrial Crops and Products. v. 34, n. 1, p. 907 $914,2011$.

PIZZI, A. Natural phenolic adhesives I: Tannin. In: PIZZI, A.; MITTAL, K. L. (Eds.) Handbook of adhesive technology. New York: Marcel Dekker, 1994, p. 573-588.

PIZZI, A. Wood adhesives: chemistry and technology. New York: Marcel Dekker, 1983. 364 p.

PIZZI, A.; MITTAL, K. L. Handbook of adhesive technology. New York: Marcel Dekker, 1994. 696 p.

ROFFAEL, E.; DIX, B. Tannine als Bindemittel für Holzwerkstoffe. Holz-Zentralblatt, v. 120, n. 6, p. 90-93, 1994.

SILVA, R. V. Uso de taninos da casca de três espécies de eucalipto na produção de adesivos para madeira. 2001. 57 p. Tese. (Mestrado em Ciência Florestal) - Universidade Federal de Viçosa, Viçosa, 2001.

VIEIRA, M. C.; LELIS, R. C. C.; SILVA, B. C.; OLIVEIRA, G. L. O. Tannin extraction from the bark of Pinus oocarpa var. oocarpa with sodium carbonate and sodium bisulfite. Floresta e Ambiente, Seropédica, v.18, n. 1, p. 1-8, jan./mar. 2011.

VITAL, B. R.; CARNEIRO, A. C. O.; PIMENTA, A. S.; LUCIA, R. M. D. Adesivos à base de taninos das cascas de duas espécies de Eucalipto para produção de chapas de flocos. Revista Àrvore, Viçosa, v. 28, n. 4, p. 571-582, 2004 .

WISSING, A. The utilization of bark II: Investigation of the Stiasny-reaction for the precipitation of polyphenols in Pine bark extractives. Svensk Papperstidning, Stockholm, v. 58, n. 20, p. 745-750, 1955.

Recebido em 08/07/2014

Aceito para publicação em 16/06/2015 